\title{
Competition for Neurotrophic Factors: Ocular Dominance Columns
}

\author{
Terry Elliott and Nigel R. Shadbolt \\ Department of Psychology, University of Nottingham, Nottingham, NG7 2RD, United Kingdom
}

Activity-dependent competition between afferents in the primary visual cortex of many mammals is a quintessential feature of neuronal development. From both experimental and theoretical perspectives, understanding the mechanisms underlying competition is a significant challenge. Recent experimental work suggests that geniculocortical afferents might compete for retrograde neurotrophic factors. We show that a mathematically well-characterized model of retrograde neurotrophic interactions, in which the afferent uptake of neurotrophic factors is activity-dependent and in which the average level of uptake determines the complexity of the axonal arbors of afferents, permits the anatomical segregation of geniculocortical afferents into ocular dominance columns. The model induces segrega- tion provided that the levels of neurotrophic factors available either by activity-independent release from cortical cells or by exogenous cortical infusion are not too high; otherwise segregation breaks down. We show that the model exhibits changes in ocular dominance column periodicity in response to changes in interocular image correlations and that the model predicts that changes in intraocular image correlations should also affect columnar periodicity.

Key words: neurotrophic interactions; ocular dominance columns; neuronal development; nerve growth factor; brainderived neurotrophic factor; competition; striate cortex; mathematical models
The segregation of geniculocortical afferents representing each eye into ocular dominance columns (ODCs) (Hubel and Wiesel, 1962) during a critical period (Hubel and Wiesel, 1970) is a common feature of the development of the primary visual cortex of many mammals (LeVay et al., 1978, 1980). Such segregation is thought to reflect the existence of activity-dependent (Reiter et al., 1986; Stryker and Harris, 1986) competitive interactions between cells from the lateral geniculate nucleus (LGN) (Guillery and Stelzner, 1970; Guillery, 1972). However, what LGN cells compete for is unclear. One possibility is that LGN cells compete for neurotrophic factors (NTFs) produced by and released from cortical cells in an activity-dependent manner (for review, see $\mathrm{Gu}$, 1995; Thoenen, 1995).

First, exogenous NTFs interfere with plasticity phenomena. Intraventricular infusion of nerve growth factor (NGF) prevents the effects of monocular deprivation (MD) in the rat LGN (Domenici et al., 1993) and visual cortex (Maffei et al., 1992; Berardi et al., 1993; Yan et al., 1996) and tempers these effects in the cat visual cortex (Carmignoto et al., 1993). Cortical application of the neurotrophin NT-4/5, but no other neurotrophin, prevents the atrophy of ferret LGN cells in response to MD (Riddle et al., 1995). In addition, cortical inf usion of either brain-derived neurotrophic factor (BDNF) or NT-4/5 prevents the formation of ODCs in the cat (Cabelli et al., 1995). Finally, blockade of the endogenous ligands of the trkB receptor (for BDNF and NT-4/5) similarly inhibits the formation of ODCs (Cabelli et al., 1997).

Second, the production and release of NTFs depend, in part, on afferent activity. For example, either dark rearing (Castren et al., 1992; Schoups et al., 1995) or MD (Bozzi et al., 1995) decreases the expression of BDNF mRNA in the rat visual cortex.

\footnotetext{
Received Feb. 9, 1998; revised May 6, 1998; accepted May 12, 1998.

This work was supported by a Royal Society University Research Fellowship to T.E.

Correspondence should be addressed to Dr. T. Elliott, Department of Psychology, University of Nottingham, Nottingham, NG7 2RD, United Kingdom.

Copyright (C) 1998 Society for Neuroscience $\quad 0270-6474 / 98 / 155850-09 \$ 05.00 / 0$
}

Although nothing is known about the release of NTFs from neurons in the visual cortex, a component in the release of BDNF (Griesbeck et al., 1995) and NGF (Blöchl and Thoenen, 1995, 1996) from hippocampal neurons does depend on afferent activity (see, also, Goodman et al., 1996).

Last, the levels of NTFs determine, in part, the complexity of afferent axonal arborizations. Although no data bear directly on the geniculocortical pathway, application of BDNF to retinotectal afferents in Xenopus increases their complexity (Cohen-Cory and Fraser, 1995). The size and complexity of axonal arborizations of sympathetic neurons in culture depend on the local availability of NGF (Campenot, 1982a,b). Also, it has recently been shown that transgenic mice expressing increased levels of BDNF in sympathetic neurons exhibit preganglionic neurons with increased axonal complexity (Causing et al., 1997).

Although NTFs also rapidly modulate synaptic efficacy in the visual cortex (Akaneya et al., 1996; Carmignoto et al., 1997), these results, taken together, are consistent with a model of competition in which geniculocortical afferents compete for a limited supply of NTFs and in which an excess supply tempers or eliminates competition (e.g., Purves, 1988). Because the local supply of NTFs affects axonal complexity, competition for NTFs leads to the local atrophy of the axonal arbors of the losing afferents and the local hypertrophy of the axonal arbors of the winning afferents, that is, anatomical segregation.

We have constructed previously a mathematical model of neurotrophic interactions (Elliott and Shadbolt, 1998). We proved that it leads to segregation provided that the exogenous supply of NTFs is below a critical value; above that value, segregation breaks down. Segregation was shown to occur even in the presence of strong correlations between the activities of afferents. These demonstrations were performed, for simplicity, in a system of two afferents and either one or two target cells. Here we generalize our study to model the segregation of geniculocortical afferents into ODCs. 


\section{MATERIALS AND METHODS}

In this section we describe the basic anatomical architecture of our model, the construction of patterns of LGN activity, the mathematical form of our model of competitive neurotrophic interactions together with a brief indication of the derivation of and the biological assumptions underlying our model, and finally the basic computational strategy and analysis of results.

We assume the LGN to consist of two $l \times l$ sheets of $l^{2}$ cells, one sheet (the "left LGN sheet") representing the left eye and the other (the "right LGN sheet") representing the right eye. The visual cortex is modeled as a $c \times c$ sheet of $c^{2}$ cells. Thus, strictly speaking, we model the formation of ocular dominance patches or domains and not columns; however, the use of the word "column" in the modeling literature is so ubiquitous that we abide by this convention. All sheets are assumed to be regular, and periodic boundary conditions are enforced for computational convenience. LGN cells are labeled by letters such as $i$ and $j$, where $i, j=1$, $\ldots, l^{2}$, and LGN sheets are labeled by Greek letters such as $\alpha$ and $\beta$, where $\alpha, \beta=L, R$, for left and right. Cortical cells are labeled by letters such as $x$ and $y$. The vector character of labels such as $i, j, x$, and $y$ is left implicit for notational convenience. Each LGN cell is constrained always to arborize over a fixed, topographically appropriate $N \times N$ square patch of cortical cells only. Initially, the arborization is approximately uniform, subject only to a small, random perturbation about perfect uniformity. The number of synapses between LGN cell $i$ in sheet $\alpha$ and cortical cell $x$ at time $t$ is denoted by $s_{x i}^{\alpha}(t)$; we will not normally indicate the time dependence explicitly. All synapses are assumed to be of fixed and equal efficacies; this is justified because we wish to model anatomical, not physiological, segregation. Of course, anatomical and physiological changes are likely to occur in concert, but because much of the data that we model here are anatomical in character (e.g., Cabelli et al., 1995), we feel justified in restricting to a model of purely anatomical plasticity. We hope, however, in later work to extend our model to include both anatomical and physiological plasticity.

We take the activity of an LGN cell to be denoted by $a_{i}^{\alpha} \in[0,1]$. To construct patterns of LGN activity, we follow closely the method used by Goodhill (1993). First, each cell $i$ in the left LGN sheet is assigned activity $a_{i}^{L}=1$ with probability 0.5 ; otherwise it is assigned activity $a_{i}^{L}=$ 0 . For each cell $i$ in the left LGN sheet, the retinotopically equivalent cell in the right LGN sheet is assigned activity $a_{i}^{R}=a_{i}^{L}$ with probability $p$; otherwise it is assigned activity $a_{i}^{R}=1-a_{i}^{L}$. The probability $p$ determines the extent of correlations between the two sets of activity patterns in the LGN sheets; $p=0$ gives perfectly anticorrelated activity patterns, $p=0.5$ gives uncorrelated activity patterns, and $p=1$ gives perfectly correlated activity patterns. We define a correlation index $C$, given by $C=2 p-1$. Finally, the activity patterns in each LGN sheet are separately convolved with a Gaussian function with parameter $\sigma_{l}$, the Gaussian being normalized appropriately on a discrete torus. The convolution serves to "smear out" the otherwise binary activity states and serves to introduce well-defined intraocular image correlations (IntraOICs) (Goodhill, 1993).

The basic evolution equation for our model of neurotrophic interactions is given by:

$$
\frac{\mathrm{d} s_{x i}^{\alpha}}{\mathrm{d} t}=\epsilon S_{x i}^{\alpha}\left[\sum_{y} \Delta_{x y}\left(T_{0}+T_{1} \frac{\sum_{\beta j} s_{y j}^{\beta} a_{j}^{\beta}}{\sum_{\beta j} s_{y j}^{\beta}}\right) \frac{\left(a+a_{i}^{\alpha}\right) \rho_{i}^{\alpha}}{\sum_{\beta j} s_{x j}^{\beta}\left(a+a_{j}^{\beta}\right) \rho_{j}^{\beta}}-1\right],
$$

where:

$$
\rho_{i}^{\alpha}=\bar{a}_{i}^{\alpha} / \sum_{x} s_{x i}^{\alpha}
$$

Here, $\epsilon$ is a parameter that determines the overall rate of change in the number of synapses, which we always assume to be $\epsilon=0.018$. We select this particular value only for consistency with previous work (Elliott and Shadbolt, 1998). Provided that it is not too large, the precise value of $\epsilon$ is unimportant (see the Appendix). The parameter $T_{0}$ represents the amount of NTF released from a cortical cell in an activity-independent manner; $T_{1}$ represents the maximum amount of NTF that can be released from a cortical cell in an activity-dependent manner; $a$ is a parameter determining the activity-independent uptake of NTF by afferents. The function $\Delta_{x y}$ characterizes the diff usion of NTF through the target field and is a function of the (minimum) Euclidean distance between the two cells $x$ and $y$ on the cortical sheet; we assume it to be a Gaussian function with parameter $\sigma_{c}$, the Gaussian being normalized appropriately on a discrete torus. Finally, the bar over $a_{i}^{\alpha}$ in the expression for $\rho_{i}^{\alpha}$ denotes the recent time average and is assumed to be given by:

$$
\bar{a}_{i}^{\alpha}(t)=\frac{1}{\tau} \int_{-\infty}^{t} \mathrm{~d} t^{\prime} a_{i}^{\alpha}\left(t^{\prime}\right) e^{-\left(t-t^{\prime}\right) / \tau}
$$

where $\tau$ is a decay constant that sets the time scale for the time average. The parameters $\epsilon$ and $\tau$ are taken to be related through $\epsilon=1 / \tau$ (see Elliott and Shadbolt, 1998, for a justification).

In brief, the ingredients and biological assumptions that go into the derivation of Equation 1 are as follows. For a full derivation, mathematical analysis, and more extensive discussion of the assumptions underlying the model, see Elliott and Shadbolt (1998).

First, we assume that the amount of NTF released by cortical cell $x$ is $r_{x}=T_{0}+T_{1} \Sigma_{\beta j} s_{x j}^{\beta} a_{j}^{\beta} / \Sigma_{\beta j} s_{x j}^{\beta} . T_{0}$ represents an activity-independent release (or, equivalently, an amount available because of the exogenous supply of NTF). The second contribution to $r_{x}$ represents an activitydependent release (Blöchl and Thoenen, 1995, 1996; Griesbeck et al., 1995; Goodman et al., 1996). The total afferent input to cortical cell $x$ is assumed to be $\Sigma_{\beta j} s_{x j}^{\beta} a_{j}^{\beta}$ must be converted into a number between 0 and 1 , inclusive, achieved by dividing by $\Sigma_{\beta j} s_{x j}^{\beta}$, so that the maximum, activity-dependent release of NTF does not exceed $T_{1}$. Other functions are possible to describe NTF release, but the form we use here is the simplest. It can be shown that the ratio $T_{0} / T_{1}$ is a key parameter determining whether afferent segregation is possible (see the Appendix for a brief derivation and Elliott and Shadbolt, 1998, for a fuller discussion).

Second, the NTF instantaneously released by cortical cells is assumed to undergo rapid diffusion through the target field, so that the amount available at each cortical cell after diffusion is assumed to be $d_{x}=\Sigma_{y} \Delta_{x y} r_{y}$. This represents the raw release of NTF from cortical cells, $r_{y}$, convolved with the diff usion function, $\Delta_{x y}$. Biologically, this amounts to the assumption that NTF diffuses from each release site independently of the diff usion from all other sites and that the amount available, after diffusion, at each site is just the sum of the amounts reaching that site by diff usion from all other sites.

Third, the uptake of NTF by afferent $i$ in LGN sheet $\alpha$ from cortical cell $x$ is assumed to be proportional to both the number of synapses $s_{x i}^{\alpha}$ and the "affinity" $\rho_{i}^{\alpha}$ of each presynaptic terminal for NTF, and the uptake is also assumed to be a function of afferent activity. (For simplicity, we assume that each terminal has precisely one active zone associated with a functional synapse; thus, we assume a one-to-one correspondence between functional synapses and anatomical presynaptic terminals.) The function of afferent activity that we use is $g_{i}^{\alpha}=a+a_{i}^{\alpha}$, where $a$ determines the resting or constitutive uptake. We assume, furthermore, that in any given period of time, the total amount of NTF available at a cortical cell is entirely exhausted by rapid afferent uptake. That NTF uptake should be proportional to the number of synapses, that is, the number of presynaptic terminals, is a reasonable assumption. This means that afferents with more synapses than other afferents in a given volume of tissue, all other factors being equal, would be at an advantage at NTF uptake. The affinity of a terminal for NTF is assumed to be proportional to the number of NTF receptors on it. Thus terminals with greater numbers of receptors are assumed to be better at taking up NTF, all other factors being equal, than are terminals with fewer numbers of receptors. The activity dependence of NTF uptake, characterized by the simple function $g$, represents a specific postulate of our model. Much experimental evidence indicates that competition between afferents depends on their relative and not their absolute levels of electrical activity (e.g., Guillery and Stelzner, 1970; Guillery, 1972). Activity-dependent NTF uptake constitutes one possible mechanism by which relative differences in electrical activity might confer a competitive advantage on more-active afferents over less-active afferents. However, we stress that we are not aware of any current experimental evidence that directly supports our hypothesis of activity-dependent NTF uptake; neither are we aware of any that directly rules it out. If the activity-independent component of NTF uptake $a$ dominates the activity-dependent component (the limit $a \rightarrow \infty$ ), then it can be shown that afferent segregation breaks down in our model (Elliott and Shadbolt, 1998).

Finally, we assume that the number of synapses $s_{x i}^{\alpha}$ from LGN cell $i$ in sheet $\alpha$ to cortical cell $x$ is equal to the recent time average of NTF uptake by LGN cell $i$ in sheet $\alpha$ from cortical cell $x$. This is a central assumption underlying our model and characterizes it as a neurotrophic model; that is, it would be difficult to justify this assumption by appealing 

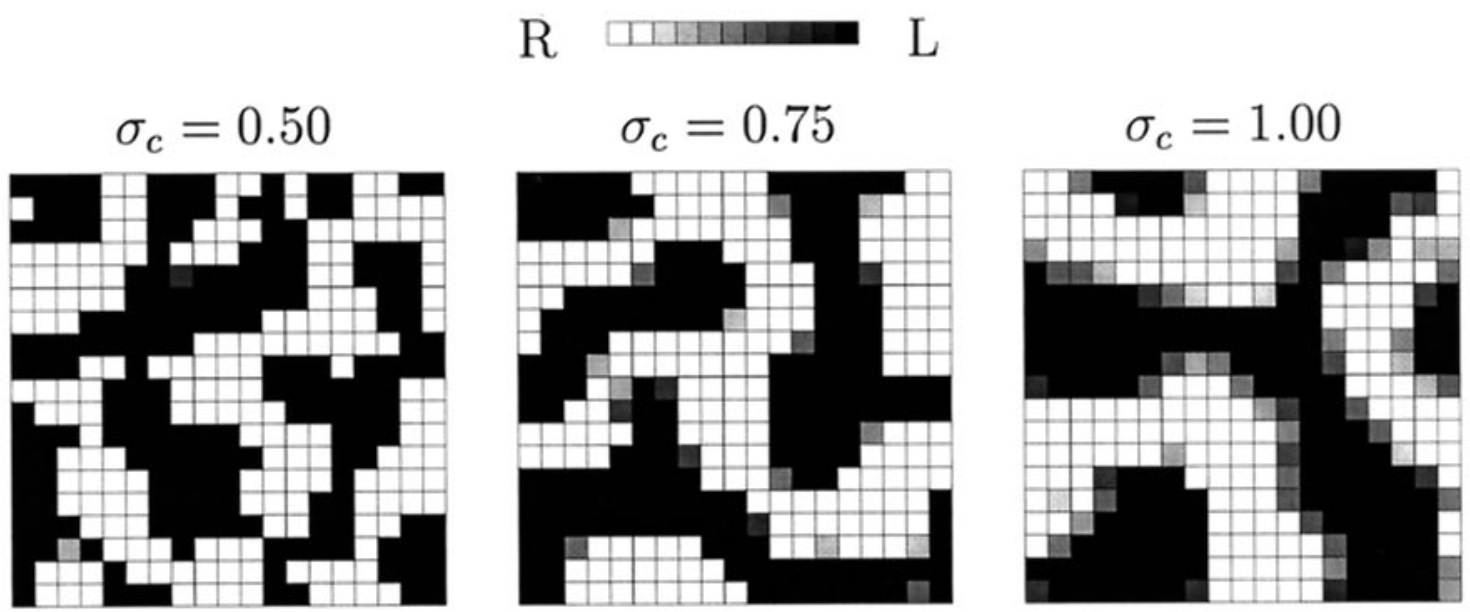

Figure 1. The final pattern of OD as a function of the NTF diffusion parameter $\sigma_{c}$. Each square in each map represents one cortical cell. The shade of gray assigned to each square represents the percentage control by the left LGN sheet; white squares are entirely controlled by the right LGN sheet ( $R$ ), and black squares are entirely controlled by the left LGN sheet $(L)$. Three cortical maps are shown, each with a value of $\sigma_{c}$ as indicated. The other parameters are $c=19, l=9, N=5, T_{0}=0, T_{1}=20, a=1, \sigma_{l}=0.75$, and $p=0.0(C=-1.0)$.

to other, non-neurotrophic aspects of synaptic plasticity. Evidence suggests that the size of the axonal arbors of afferents is a function of neurotrophic support (Cohen-Cory and Fraser, 1995; Causing et al., 1997). Furthermore, the local influence of NTF level on the local size of axonal arbors required by our assumption seems to be justified by the observation that local NGF shortage or excess supply results in the local atrophy or hypertrophy, respectively, of that part of the axonal arbor experiencing the shortage or excess supply (Campenot, 1982a,b).

These assumptions lead directly to Equation 1, where Equation 2 constitutes a particular model for affinity. This model requires that the number of NTF receptors per terminal depends on the average level of afferent electrical activity. Support for this requirement comes from the fact that kindling or seizures in the rat result in an increase in the mRNAs for trkB and trkC, the non-NGF neurotrophin high-affinity receptors (Bengzon et al., 1993; Dugich-Djordjevic et al., 1995; Salin et al., 1995), as do depolarizing media (Birren et al., 1992; Cohen-Cory et al., 1993). The particular model of affinity also requires that the number of NTF receptors per terminal is inversely proportional to the number of terminals. No experimental data bear directly on this requirement, but recent work on the Drosophila neuromuscular junction indicates that certain mutants exhibit motor neurons with approximately twice as many synaptic boutons as controls (Schuster et al., 1996a,b). However, the synaptic efficacy of such mutant motor neurons is unchanged, suggesting that the same level of synaptic machinery is simply distributed over a larger axonal arbor (Schuster et al., 1996a,b). Thus, it is conceivable that NTF receptors might also be redistributed around an axonal arbor in response to anatomical plasticity. In any event, some presynaptic mechanism seems to be necessary to prevent an afferent from retracting all of its terminals during ODC development, and changes in NTF receptors are an appealing candidate within the framework of a neurotrophic model. Another possibility, different from the one used here, although perhaps broadly similar in terms of developmental outcome, is that NTF receptors might increase (decrease) their sensitivity to NTFs as an axonal arbor undergoes atrophy (hypertrophy). For the purposes of simplicity and, to some degree, mathematical tractability, we examine only the first-mentioned possibility, that of an inverse relationship between the number of receptors per terminal and the total number of terminals.

Our basic computational strategy is straightforward. We construct an initial, approximately uniform innervation of the cortex by the LGN. Then a pattern of LGN activity is generated, and Equation 1 is used to modify the number of synapses between an afferent and a target cell. Because the number of synapses must be an integer, but Equation 1 is a continuous equation, after each application of Equation 1, we discretize the $s_{x i}^{\alpha}$. In fact, $T_{1}$ sets the scale for the $s_{x i}^{\alpha}$ and is essentially arbitrary. We simply set $T_{1}=20$ for convenience. The discretization of the $s_{x i}^{\alpha}$ is then achieved by converting $100 s_{x i}^{\alpha}$ to the nearest integer, so that $s_{x i}^{\alpha}$ grows or decays in steps of 0.01 . Equivalently, we could set $T_{1}=2000$ and simply convert $s_{x i}^{\alpha}$ to the nearest integer, but numerically it is safer to use numbers of order of approximately unity. With these choices, each cortical cell then supports $\sim 1000$ geniculocortical synapses. The overall process of LGN activation and updating of the number of synapses is typically repeated $5.0 \times 10^{5}$ or $1.0 \times 10^{6}$ times, unless otherwise indicated.

Finally, because we will be interested in the periodicity of the resulting patterns of ocular dominance (OD) produced by our simulations, we calculate the two-dimensional Fourier transforms of the overall patterns. OD is quantified as the percentage control of a cortical cell by the left LGN sheet. To calculate the Fourier transform, we rescale this percentage control into a number in the interval $[-1,+1]$, with -1 representing control by the right LGN sheet, 0 representing equal control by both sheets, and +1 representing control by the left LGN sheet. Having determined the Fourier transform of such a rescaled pattern, we extract the power spectrum. The peak of the power spectrum determines the dominant spatial frequency present in the pattern.

\section{RESULTS}

We now present results of simulations of our model of neurotrophic interactions applied to the ODC system. We set $c$, the size of the cortex, to 19 ; $l$, the size of the LGN sheets, to 9; and $N$, the size of the patch of cortex over which LGN cells arborize, to 5 . There is little qualitative change in our results for other selections of these parameters except for $N$, whose role we discuss below. We also set $T_{1}=20$, as mentioned earlier, and unless otherwise indicated, we assume $T_{0}=0$. This latter means that there is typically no activity-independent release of NTF from cortical cells, or no exogenous infusion of NTF into the cortex. We also assume $a=1$, which determines an activity-independent component in the uptake of NTF by afferents (see Elliott and Shadbolt, 1998 , for a discussion of the role of the parameter $a$ in segregation). Unless otherwise stated, we will assume $p=0(C=-1)$, so that the patterns of activity in the two LGN sheets are perfectly anticorrelated; $\sigma_{l}$, which determines the extent of IntraOICs, will be set to 0.75 ; and $\sigma_{c}$, which determines the extent of diff usion of NTF through the target field, will be set to 0.75 . We will explore the effect of changes in these parameters on the overall pattern of OD. For a brief, partial analysis of the influence of these parameters, particularly $p$, on the overall pattern of OD, see the Appendix.

In Figure 1, we show the final pattern of OD for three different values of $\sigma_{c}$, and Figure 2 shows the corresponding power spectra. As $\sigma_{c}$ increases, the number of binocularly controlled cells in the 


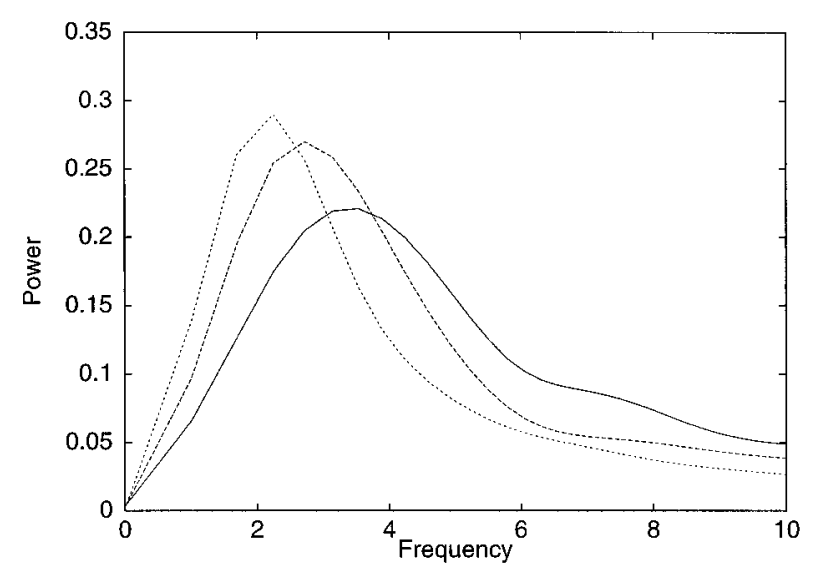

Figure 2. The power spectra corresponding to the three maps in Figure 1 . The solid line represents $\sigma_{c}=0.50$, the long-dashed line represents $\sigma_{c}=$ 0.75 , and the short-dashed line represents $\sigma_{c}=1.00$.

final patterns increases. This occurs because larger values of $\sigma_{c}$, which correspond to more extensive diff usion of NTF, permit the integration of responses over larger retinotopic separations. However, larger retinotopic separations are associated with reduced IntraOICs, leading to a weakening of co-operation between LGN cells in the same LGN sheet, and thus increased binocularity. In addition, as $\sigma_{c}$ increases, the width of ODCs increases, as shown by the decrease in the peak frequencies of the power spectra in Figure 2. This behavior is a common feature of a wide range of models of ODC formation (e.g., Swindale, 1980; Miller et al., 1989; Goodhill, 1993). The parameter $N$, which sets the size of the arborization patch for LGN axons, sets an upper bound on column width; for $\sigma_{c}=1.00$, column width is approximately saturated, with no further increases being possible.

Figure 3 shows the effect of changing the extent of IntraOICs by varying $\sigma_{l}$, and Figure 4 shows the associated power spectra. In contrast to increasing $\sigma_{c}$, increasing $\sigma_{l}$ decreases the number of binocularly controlled cells in the final patterns. This occurs because co-operation between LGN cells from the same sheet increases, thereby increasing competition with cells from the other LGN sheet. An increase in $\sigma_{l}$ is also associated with an increase in the width of ODCs, with, again, an upper limit on width set by $N$. This shift in ODC width in response to changes in IntraOICs is also observed in another of our models of retrograde neurotrophic interactions (Elliott et al., 1996; T. Elliott and N. Shadbolt, unpublished observations). It would therefore seem to be a reliable result.

The effect of changing interocular image correlations (InterOICs), rather than IntraOICs, measured by the correlation index $C$, is shown in Figure 5, with the power spectra shown in Figure 6. As InterOICs decrease, as in the strabismic rearing of kittens, afferent segregation is enhanced (Hubel and Wiesel, 1965; Shatz et al., 1977). Furthermore, ODC width increases as $C$ decreases. This is a result that was first predicted in the biologically motivated model of Goodhill (1993), although it was predicted earlier in a biologically abstract model (Goodhill and Willshaw, 1990). This prediction has recently been confirmed experimentally (Löwel, 1994; Goodhill and Löwel, 1995; see also Tieman and Tumosa, 1997).

So far we have shown that a model of retrograde neurotrophic interactions can lead to the activity-dependent segregation of geniculocortical afferents and can also account for much experimental data. However, one of the criteria that our model must satisfy for it to be a viable model of neurotrophic interactions in the visual cortex is that the simulated infusion of NTF into the developing visual cortex must lead to a breakdown of anatomical segregation (Cabelli et al., 1995). To this end, we take a simulated cortex in which afferent segregation is underway, but not complete, and simulate the infusion of NTF by setting $T_{0}=100$. This is shown in Figure 7. We see that infusion of NTF reverses the partial segregation. This is achieved by the exogenously applied NTF promoting nonspecific afferent sprouting and not by stabilizing synapses, which would merely freeze the initial, preinfusion pattern of ocular dominance.

\section{DISCUSSION}

We have shown that a model of activity-dependent competition between geniculocortical afferents based on retrograde neurotrophic interactions robustly leads to the development of ODCs. This extends our previous characterization of the model (Elliott and Shadbolt, 1998). We have demonstrated that the model accounts for several experimental results, including the break-
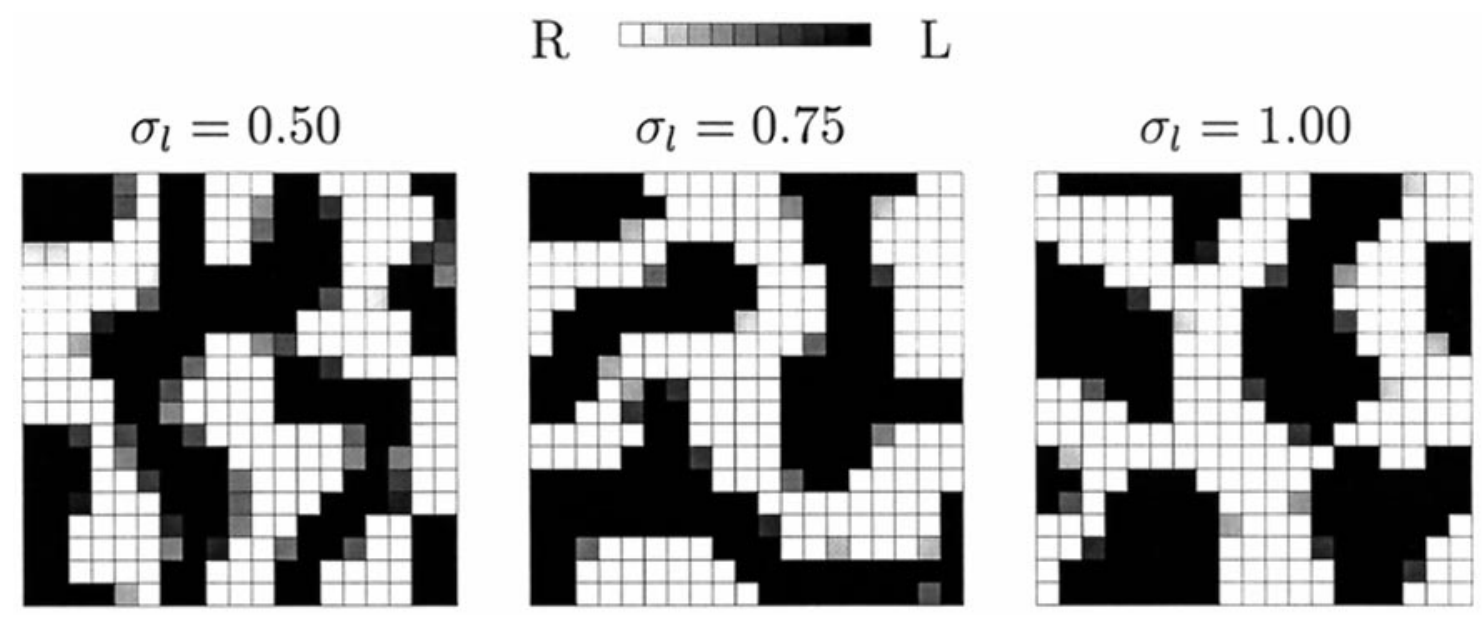

Figure 3. The final pattern of OD as a function of the intraocular image correlation parameter $\sigma_{l}$. Three cortical maps are shown, each with a value of $\sigma_{l}$ as indicated. The other parameters are $c=19, l=9, N=5, T_{0}=0, T_{1}=20, a=1, \sigma_{c}=0.75$, and $p=0.0(C=-1.0)$. 


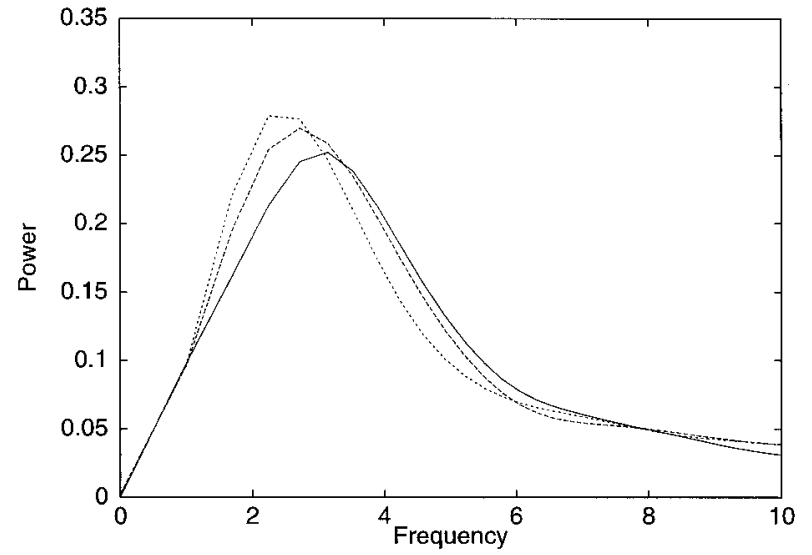

Figure 4. The power spectra corresponding to the three maps in Figure 3. The solid line represents $\sigma_{l}=0.50$, the long-dashed line represents $\sigma_{l}=$ 0.75 , and the short-dashed line represents $\sigma_{l}=1.00$.

down of anatomical segregation after cortical infusion of either BDNF or NT-4/5 (Cabelli et al., 1995).

A striking result exhibited by our model is the dependence of ODC width on the correlation index $C$ (Figs. 5, 6). This dependence was predicted by a biologically realistic model (Goodhill, 1993) before the experimental discovery of the fact that strabismus increases the width of ODCs in kittens (Löwel, 1994; Goodhill and Löwel, 1995; see also Tieman and Tumosa, 1997). No other biologically realistic models have so far accounted for such a change. Goodhill's model was constructed to account not only for the development of ODCs but also for the refinement of an initially coarse topographic projection (Goodhill, 1993). To achieve the latter, Goodhill does not use an arbor function, as here, but instead allows each LGN cell to arborize over every cortical cell, while including a slight topographic bias in the initial projections. Furthermore, Goodhill uses a "winner-take-all" cortical activation function, in which intracortical dynamics are assumed to be such that inhibition silences all cortical cells except those in the neighborhood of the cortical cell receiving the largest synaptic strength-weighted LGN input.

However, the results presented by Goodhill (1993) do not unequivocally determine precisely which aspects of his model allow ODC width to depend on the correlation index $C$. One possibility is the absence of an arbor constraint. However, our results rule this out because our model uses an arbor constraint but ODC width still depends on $C$. It has been claimed that the nonlinear cortical activation dynamics implicit in the winnertake-all rule are responsible and that if such were introduced into otherwise linear models (Miller et al., 1989), then these models would also exhibit a dependence of ODC width on $C$ (Miller, 1995). Again, our results clearly eliminate this because our model does not use a winner-take-all rule. Indeed, under suitable reinterpretations, our intracortical diffusion function is equivalent to an excitatory intracortical function with no inhibition. Although our model is nonlinear, the linear analysis performed in the Appendix indicates that the correlation index $C$ can affect the largest spatial frequency in the final pattern of OD. These considerations thus eliminate, on the grounds of incompatibility with experimental results, linear, correlation-based models and simple modifications thereof, such as that of Miller et al. (1989).

In addition to depending on InterOICs, our model predicts that ODC width also depends on IntraOICs. This is a result observed in another, much simpler neurotrophic model (Elliott et al., 1996; Elliot and Shadbolt, unpublished observations). This prediction would thus seem to be quite robust and therefore worth experimental investigation. However, it is likely to be difficult to test. One way of doing so would be to stimulate the optic nerves directly (Stryker and Strickland, 1984; Weliky and Katz, 1997), while carefully controlling the extent of InterOICs to avoid reversing any increase in column width attributable to increased IntraOICs by inducing an associated decrease in column width attributable to increased InterOICs (contrast Figs. 3, 5). Another, perhaps easier manipulation might be either to blur the retinal image or else to enlarge it with suitably designed contact lenses. Simple, correlation-based models do not exhibit a dependence of ODC width on IntraOICs (as well as InterOICs) (Miller et al., 1989), so our prediction constitutes another way of distinguishing between those models and ours.

Our model is constructed in an attempt to understand the biological mechanisms underlying activity-dependent, competitive interactions. Our results (Fig. 5) demonstrate that it can robustly segregate afferents in the presence of positively correlated interocular images, such as presumably occur during normal
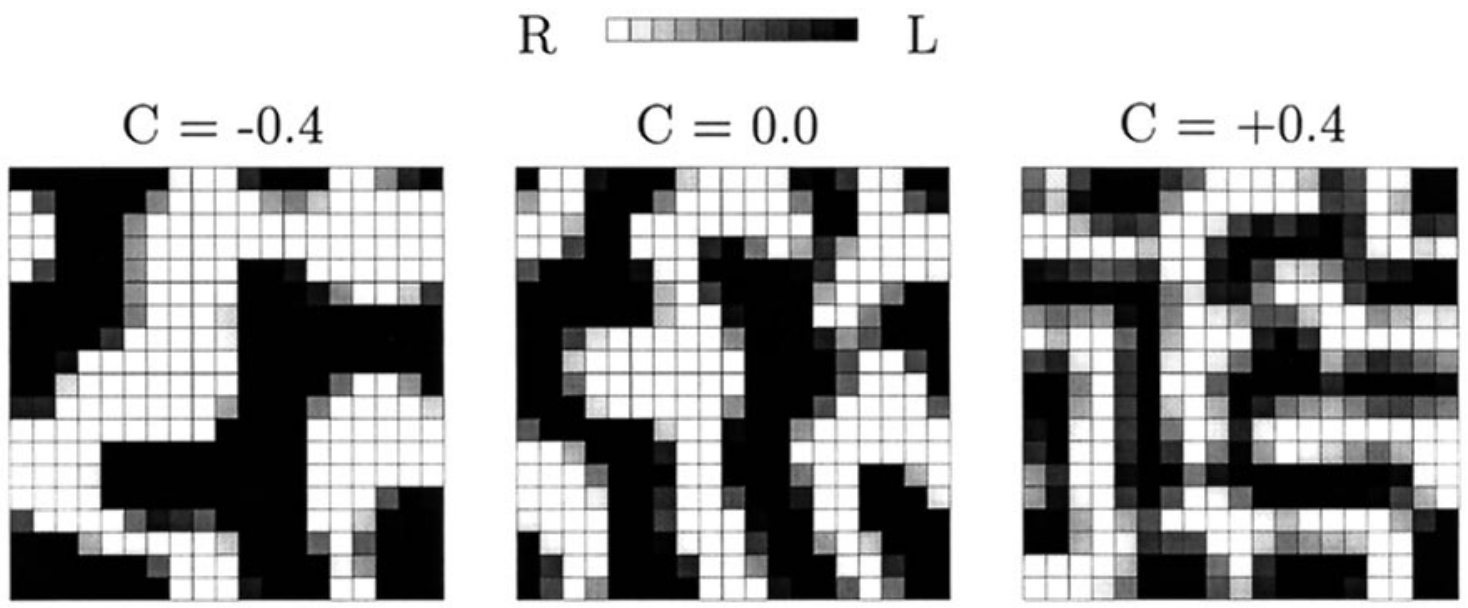

Figure 5. The final pattern of OD as a function of the interocular image correlation parameter $C$. Three cortical maps are shown, each with a value of $C=2 p-1$ as indicated. The other parameters are $c=19, l=9, N=5, T_{0}=0, T_{1}=20, a=1, \sigma_{c}=0.75$, and $\sigma_{l}=0.75$. 


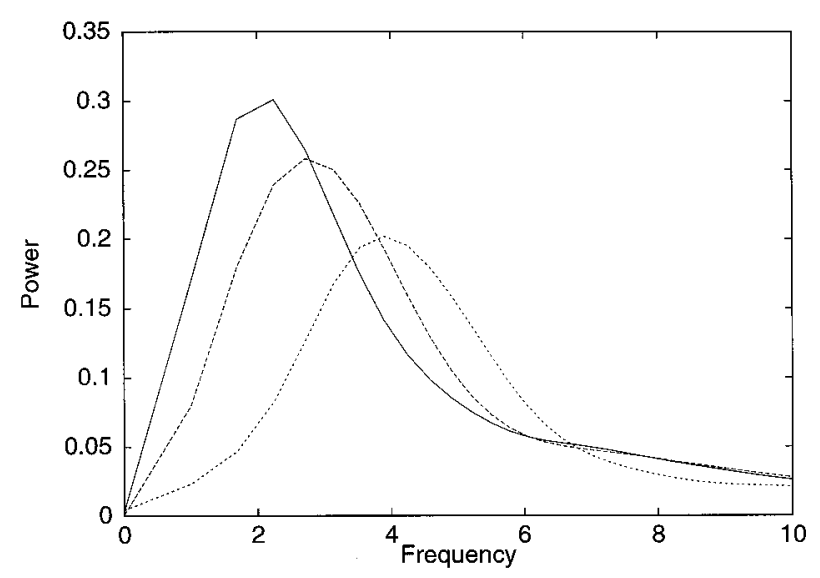

Figure 6. The power spectra corresponding to the three maps in Figure 5. The solid line represents $C=-0.4$, the long-dashed line represents $C=$ 0.0 , and the short-dashed line represents $C=+0.4$.

vision in the kitten. Models of ODC formation have, however, typically used subtractive postsynaptic normalization, in which the summed physiological strengths of synapses projected to cortical cells are constrained to be constant, to segregate afferents in such a case (Miller et al., 1989; Goodhill, 1993). However, normalization is unsatisfactory because it merely enforces rather than attempts to illuminate the quintessential feature of development in the visual cortex, namely competition, and furthermore, there is little experimental evidence in support of normalization (for alternatives to normalization, see, for example, Bienenstock et al., 1982; Montague et al., 1991; Elliott et al., 1996).

Very recent experimental evidence, however, does seem to support the possibility of multiplicative postsynaptic normalization (Turrigiano et al., 1998). This evidence is obtained in a cortical culture system under the arguably pathological regimes of either total activity blockade (using tetrodotoxin) or blockade of inhibition (using bicuculline). Whether this result generalizes to the in vivo situation and, in particular, to normal, physiological activity regimes is presently unclear. Despite this possibility, multiplicative postsynaptic normalization is an inadequate candidate for inducing competition between geniculocortical afferents, because it is well known that this form of normalization cannot induce the segregation of afferents in the presence of positive image correlations between the two eyes.

For segregation to be possible in the presence of positive correlations, subtractive postsynaptic normalization must be used (Miller et al., 1989; Goodhill, 1993). However, subtractive normalization is particularly problematic, because it requires that the assumed decay of synaptic strengths that is postulated to bring about normalization is independent of the concentrations of any of the substances that contribute to the physiological efficacy of a synapse in inducing a depolarization of the postsynaptic membrane. By proposing, in contrast, a neurotrophic model in which the uptake of NTF depends, in part, on afferent activity and in which the time-averaged NTF uptake determines the local complexity of the arbors of afferents, we have shown that competitive interactions emerge naturally. To that extent, our model proposes concrete, testable hypotheses concerning the mechanisms underlying activity-dependent competition in the visual cortex.

A class of experimental results that we have not attempted to model here is that associated with MD. In our model, MD would lead to the deprived-eye (undeprived-eye) afferents being less

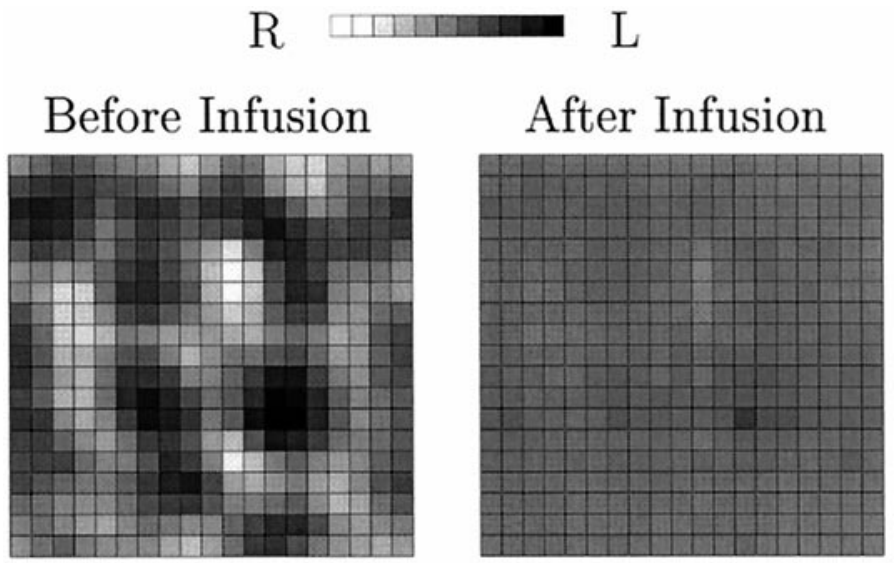

Figure 7. The simulated infusion of NTF into the visual cortex in which geniculocortical afferents have partially but not completely segregated. Left, Map that is the result of 20,000 presentations of LGN activity patterns and has $T_{0}=0$. Right, Map generated by setting $T_{0}=100$ and running the simulation for a further 30,000 presentations of LGN activity patterns. The other parameters are $c=19, l=9, N=5, T_{1}=20, a=1$, $\sigma_{c}=0.75, \sigma_{l}=0.75$, and $p=0.0(C=-1.0)$.

(more) advantaged in taking up NTF. This would lead to the atrophy (hypertrophy) of the axonal arbors of the deprived-eye (undeprived-eye) afferents. Our model thus accounts for the basic phenomenology of MD. However, there are a number of experimental results that either indicate that our model requires further extension or are generally conf using.

First, cortical infusion of the GABA receptor $\left(\mathrm{GABA}_{\mathrm{A}}\right)$ agonist muscimol results in a paradoxical shift of OD toward the deprived eye (Reiter and Stryker, 1988; Hata and Stryker, 1994). Muscimol infusion is likely to cause a generalized decrease in the cortical cell production and release of NTFs (Zafra et al., 1991; Blöchl and Thoenen, 1995, 1996; Griesbeck et al., 1995). Furthermore, much evidence suggests that electrical activity exerts a regressive influence on afferents (e.g., Cohan and Kater, 1986; Sussdorf and Campenot, 1986; Lipton and Kater, 1989; Mattson and Kater, 1989; Fields et al., 1990). Thus, axonal complexity appears to be influenced both by NTF-induced growth (e.g., Campenot, 1982a,b; Cohen-Cory and Fraser, 1995; Causing et al., 1997) and by afferent activity-induced retraction. The generalized reduction in NTFs that probably results from muscimol infusion might therefore shift the balance between these two antagonistic tendencies in favor of retraction. Hence, moreactive, undeprived afferents should retract, resulting in the passive expansion of the less-active, deprived afferents. Inclusion of such regressive influences in the present model should be straightforward (cf. Elliott and Shadbolt, 1996). It is also possible that muscimol infusion interferes with inhibitory circuitry.

Second, although intraventricular infusion of NGF prevents or tempers the response to MD (Maffei et al., 1992; Berardi et al., 1993; Carmignoto et al., 1993; Domenici et al., 1993; Yan et al., 1996), cortical infusion of NGF is without effect, whereas BDNF results in paradoxical OD shifts (Galuske et al., 1996). Cortical NGF infusion in adult cats reinstates OD plasticity, but the shift is paradoxical (Gu et al., 1994). However, cortical application of NT-4/5, but no other neurotrophin, does prevent the atrophy of deprived-eye-controlled LGN cell bodies in young ferrets (Riddle et al., 1995). In view of the possibly contradictory nature of some of these results, we adopt the position that, in the absence of definitive evidence to the contrary, the general neurotrophic 
thesis that an excess supply of NTFs should temper or eliminate competition, and thus temper or prevent a response to $\mathrm{MD}$, remains intact.

Recently a very interesting neurotrophic model of the development of ocular dominance columns has appeared (Harris et al., 1997). However, this model seems to possess a number of difficulties. First, it considers only physiological plasticity and disregards anatomical plasticity; yet the evidence of the breakdown of segregation of LGN afferents after exogenous infusion of NTFs is anatomical, not physiological (Cabelli et al., 1995). In addition, the model assumes a constant pool of available NTF, so that NTF production and release is not regulated by neuronal activity, in contrast to the experimental situation (Castren et al., 1992; Bozzi et al., 1995; Schoups et al., 1995). Finally, the model makes the rather implausible assumption that the uptake of NTF by afferents depends on the synaptic efficacy of afferents.

In conclusion, we have shown that a model of neurotrophic interactions based on the ideas that the uptake of NTFs is activity-dependent and that the time-averaged level of uptake determines the local complexity of arbors of afferents leads to activity-dependent competition between geniculocortical afferents and thus to their segregation. We have shown that the model exhibits a dependence of ODC width on InterOICs and that the model also predicts that IntraOICs affect ODC width.

\section{APPENDIX}

Here we show first that ODC width exhibits a dependence on the parameter $C$, which determines the extent of InterOICs. We then briefly indicate some of the results of a fixed-point analysis. This demonstrates that the ratio $T_{0} / T_{1}$ plays a crucial role in determining whether afferent segregation is possible. It also shows that the value of $\epsilon$ is essentially arbitrary.

To determine how the parameter $C$ affects ODC width, we need to analyze Equation 1. Ideally, to perform an analysis of Equation 1, we would like to average it over all patterns of LGN activity, assuming that the $s_{x i}^{\alpha}$ changes sufficiently slowly. However, the highly nonlinear character of the equation prevents general, direct averaging. One way in which to avoid this problem is to assume that all cells in an LGN sheet have the same activities, that is, $a_{i}^{\alpha}=a^{\alpha} \forall i, \alpha$. The risk in doing this, as Figures 3 and 4 indicate, is that of irreversibly saturating ODC width at the maximum permitted value of $N$. For many choices of parameters, this is indeed the case. However, in some regions of parameter space, in which $\sigma_{c}$ is small, changes in ODC width are observable. Thus, with the assumption that all cells within an LGN sheet have the same activities and writing $c=T_{0} /\left(a T_{1}\right)$, Equation 1 becomes:

$$
\frac{\mathrm{d} s_{x i}^{\alpha}}{\mathrm{d} t}=\epsilon S_{x i}^{\alpha}\left[T_{1} \sum_{y} \Delta_{x y} \frac{\sum_{\beta}\left(a c+a^{\beta}\right) \sum_{j} s_{y j}^{\beta}}{\sum_{\beta j} s_{y j}^{\beta}} \frac{\left(a+a^{\alpha}\right) \rho_{i}^{\alpha}}{\sum_{\beta}\left(a+a^{\beta}\right) \sum_{j} s_{y j}^{\beta} \rho_{j}^{\beta}}-1\right] .
$$

The initial conditions are:

$$
s_{x i}^{\alpha}=\frac{1}{2 N^{2}} T_{1}\left(a c+\frac{1}{2}\right) A_{x i}+\delta_{x i}^{\alpha}
$$

where we have assumed that the average activity of each afferent is:

$$
\bar{a}_{i}^{\alpha}=\frac{1}{2} \forall i, \alpha,
$$

and the function $A_{x i}$ is an arbor function such that $A_{x i}=1$ if, and only if, cortical cell $x$ is within the cortical patch over which LGN cells at retinotopic position $i$ arborize and zero otherwise. The function $\delta_{x i}^{\alpha}$ represents a small perturbation about an initial, uniform innervation of the cortex by LGN cells; outside the arbor region, it is assumed to vanish, that is, $\delta_{x i}^{\alpha}=0$ when $A_{x i}=0$.

To determine the width of ODCs, we linearize Equation 4 in the $\delta_{x i}^{\alpha}$ The linearized equations are then explicitly averaged over all possible values of $a^{\alpha}$. To perform the averaging, we assume for simplicity that $a^{\alpha} \in\{0,1\}, \alpha=L, R$, and we assume an unbiased distribution of "on" $\left(a^{\alpha}=1\right)$ and "off" $\left(a^{\alpha}=0\right)$ states. Defining $\delta_{x i}^{ \pm}=\delta_{x i}^{L} \pm \delta_{x i}^{R}$ and $\delta_{x}^{ \pm}=\Sigma_{i} \delta_{x i}^{ \pm}$and after much algebra, we obtain:

$$
\frac{\mathrm{d}}{\mathrm{d} t}\left\langle\delta_{x}^{+}\right\rangle=-\epsilon\left\langle\delta_{x}^{+}\right\rangle
$$

$\frac{\mathrm{d}}{\mathrm{d} t}\left\langle\delta_{x}^{-}\right\rangle=\epsilon q\left[N^{-2}\left(B-q^{-1}\right) \sum_{y j} A_{x j}\left\langle\delta_{y j}^{-}\right\rangle+\sum_{y}\left(A \Delta_{x y}-B \delta_{x y}\right)\left\langle\delta_{y}^{-}\right\rangle\right]$,

where \langle\rangle denotes the averaging over LGN activity, $q=1-p$, and the constants $A$ and $B$ are given by $A^{-1}=(2 a+1)(2 a c+1)$ and $B^{-1}=(2 a+1)^{2}$.

Equation 7 describes the early development of ODCs. The first term on the right-hand side (RHS) of this equation arises directly from the presence of the $\rho_{i}^{\alpha}$ terms in Equation 4; if $\rho_{i}^{\alpha}=1 \forall i, \alpha$, then this term disappears. In the absence of this term, the Fourier transform of Equation 7 indicates that different spatial frequencies decouple and that the fastest-growing spatial frequency is that which, in Fourier space, maximizes the RHS. This fastestgrowing component is likely to dominate the final pattern of OD and thus gives the width of ODCs (e.g., Swindale, 1980; Miller et al., 1989). Furthermore, the extent of InterOICs does not affect the final pattern of OD but simply slows down its development, effectively by reducing the value of $\epsilon$.

In the presence of the first term on the RHS of Equation 7, however, matters are more complicated. This is because it cannot be expressed simply in terms of the variables $\delta_{x}^{-}$. Therefore the initial evolution of these variables depends in part on the precise patterns of connectivity between individual LGN cells and individual cortical cells rather than just the connectivity between whole LGN sheets and individual cortical cells. However, this first term indicates that both the arbor function $A_{x i}$ and the extent of InterOICs $q$ affect the development of different spatial frequencies. Although further analysis of this equation is necessary (which we do not perform here), these preliminary observations at least give a partial, analytical basis to the observed dependence of ODC width on the correlation index $C=2 p-1=1-2 q$.

We now turn to a brief indication of the results of a fixed-point analysis. To facilitate analysis, we restrict the analysis to two afferent cells, one in each of the two LGN sheets, and two target cells and ignore diffusion of NTF between target cells (so that $\left.\Delta_{x y}=\delta_{x y}\right)$. This means that $i=1$ only and $x=1,2$ only. Each cell in each LGN sheet will be assumed to arborize over both cortical cells (so that $A_{x 1}=1$ for $x=1,2$ ). Thus the arbor region is no longer a square, but Equation 7 is still valid because the factor of $N^{2}$ appearing on the RHS refers not to the particular geometry of innervation but only to the number of target cells innervated. Therefore, we need only assume $N^{2}=2$.

Equations 6 and 7 show that the point defined by Equation 5 with $\delta_{x i}^{\alpha}=0$ is a fixed point of the afferent activity-averaged system. To determine the stability of this fixed point, we need to obtain the eigenvalues of the evolution matrix of the linearized 
system. Two of these eigenvalues, for $\left\langle\delta_{x}^{+}\right\rangle, x=1,2$, are given immediately by Equation 6; namely, they are both $-\epsilon$. The other two eigenvalues are the eigenvalues of the matrix in the equation:

$$
\frac{\mathrm{d}}{\mathrm{d} t}\left(\begin{array}{c}
\left\langle\delta_{1}^{-}\right\rangle \\
\left\langle\delta_{2}^{-}\right\rangle
\end{array}\right)=\frac{\epsilon}{2}\left(\begin{array}{cc}
q(2 A-B)-1 & q B-1 \\
q B-1 & q(2 A-B)-1
\end{array}\right)\left(\begin{array}{l}
\left\langle\delta_{1}^{-}\right\rangle \\
\left\langle\delta_{2}^{-}\right\rangle
\end{array}\right),
$$

where we have rewritten Equation 7 in matrix form for target cells $x=1,2$. The eigenvalues of this matrix can be read off by inspection. One is $\epsilon(q A-1)$, which is negative-semidefinite (i.e., never positive) because $0 \leq q A \leq 1$, and the other reduces to:

$$
\epsilon \frac{2 a(1-c) q}{(2 a+1)^{2}(2 a c+1)}
$$

This eigenvalue changes sign at $c=T_{0} /\left(a T_{1}\right)=1$, that is, when $T_{0} / T_{1}=a$. Thus, if $c>1$, then all eigenvalues are negative, and the fixed point is stable; but if $c<1$, then one eigenvalue is positive, and the fixed point turns into a saddle. This means that for $c<1$, the unsegregated state is unstable, but for $c>1$, it is stable.

Two other fixed points of the two-afferent, two-target cell system exist, corresponding to completely segregated states (one for each of the two possible, opposite states of segregation), and their stability is exactly the reverse of the fixed point corresponding to the unsegregated state considered above. Thus, for $c<1$, these additional fixed points are stable, whereas for $c>1$, they are unstable. Hence, the ratio $T_{0} / T_{1}$ critically determines whether or not segregation can occur.

All of the eigenvalues of the matrices corresponding to the various fixed points depend linearly on $\epsilon$. Thus, the value of $\epsilon$, in the afferent activity-averaged system, determines the rate at which the system moves toward or away from the fixed points. Its value does not change the behavior of the system in any other way. Thus, in the nonaveraged system, it suffices that $\epsilon$ is sufficiently small to make the system behave like an afferent activityaveraged system. To that extent, its values are essentially arbitrary.

\section{REFERENCES}

Akaneya Y, Tsumoto T, Hatanaka H (1996) Brain-derived neurotrophic factor blocks long-term depression in the rat visual cortex. J Neurophysiol 76:4198-4201.

Bengzon J, Kokaia Z, Ernfors P, Kokaia M, Leanza G, Nilsson OG, Persson H, Lindvall O (1993) Regulation of neurotrophin and trkA, trkB and trkC tyrosine kinase receptor mRNA expression in kindling. Neuroscience 53:433-446.

Berardi N, Domenici L, Parisi V, Pizzorusso T, Cellerino A, Maffei L (1993) Monocular deprivation effects in the rat visual cortex and lateral geniculate nucleus are prevented by nerve growth factor (NGF). I. Visual cortex. Proc R Soc Lond [Biol] 251:17-23.

Bienenstock EL, Cooper LN, Munro PW (1982) Theory for the development of neuron selectivity: orientation specificity and binocular interaction in visual cortex. J Neurosci 2:32-48.

Birren SJ, Verdi JM, Anderson DJ (1992) Membrane depolarisation induces $\mathrm{p} 140^{\text {trk }}$ and NGF responsiveness, but not $\mathrm{p} 75^{\mathrm{LNGFR}}$, in MAH cells. Science 257:395-397.

Blöchl A, Thoenen H (1995) Characterization of nerve growth factor (NGF) release from hippocampal neurons: evidence for a constitutive and an unconventional sodium-dependent regulated pathway. Eur J Neurosci 7:1220-1228.

Blöchl A, Thoenen H (1996) Localization of cellular storage compartments and sites of constitutive and activity-dependent release of nerve growth factor (NGF) in primary cultures of hippocampal neurons. Mol Cell Neurosci 7:173-190.

Bozzi Y, Pizzorusso T, Cremisi F, Rossi FM, Barsacchi G, Maffei L (1995) Monocular deprivation decreases the expression of messenger
RNA for brain-derived neurotrophic factor in the rat visual cortex. Neuroscience 69:1133-1144.

Cabelli RJ, Hohn A, Shatz CJ (1995) Inhibition of ocular dominance column formation by infusion of NT-4/5 or BDNF. Science 267:1662-1666.

Cabelli RJ, Shelton DL, Segal RA, Shatz CJ (1997) Blockade of endogenous ligands of trkB inhibits formation of ocular dominance columns. Neuron 19:63-76.

Campenot RB (1982a) Development of sympathetic neurons in compartmentalized cultures. I. Local control of neurite outgrowth by nerve growth factor. Dev Biol 93:1-12.

Campenot RB (1982b) Development of sympathetic neurons in compartmentalized cultures. II. Local control of neurite survival by nerve growth factor. Dev Biol 93:13-22.

Carmignoto G, Canella R, Candeo P, Comelli MC, Maffei L (1993) Effects of nerve growth factor on neuronal plasticity of the kitten visual cortex. J Physiol (Lond) 464:343-360.

Carmignoto G, Pizzorusso T, Tia S, Vicini S (1997) Brain-derived neurotrophic factor and nerve growth factor potentiate excitatory synaptic transmission in the rat visual cortex. J Physiol (Lond) 498:153-164.

Castren E, Zafra F, Thoenen H, Lindholm D (1992) Light regulates expression of brain-derived neurotrophic factor mRNA in rat visual cortex. Proc Natl Acad Sci USA 89:9444-9448.

Causing CG, Gloster A, Aloyz R, Bamji SX, Chang E, Fawcett J, Kuchel G, Miller FD (1997) Synaptic innervation density is regulated by neuron-derived BDNF. Neuron 18:257-267.

Cohan CS, Kater SB (1986) Suppression of neurite elongation and growth cone motility by electrical activity. Science 232:1638-1640.

Cohen-Cory S, Fraser SE (1995) Effects of brain-derived neurotrophic factor on optic axon branching and remodelling in vivo. Nature 378:192-196.

Cohen-Cory S, Elliott RC, Dreyfus CF, Black IB (1993) Depolarizing influences increase low affinity NGF receptor gene expression in cultured Purkinje neurons. Exp Neurol 119:165-173.

Domenici L, Cellerino A, Maffei L (1993) Monocular deprivation effects in the rat visual cortex and lateral geniculate nucleus are prevented by nerve growth factor (NGF). II. Lateral geniculate nucleus. Proc R Soc Lond [Biol] 251:25-31.

Dugich-Djordjevic MM, Ohsawa F, Okazaki T, Mori N, Day JR, Beck KD, Hefti F (1995) Differential regulation of catalytic and noncatalytic trkB mRNAs in the rat hippocampus following seizures induced by systemic administration of kainate. Neuroscience 66:861-877.

Elliott T, Shadbolt NR (1996) A mathematical model of activitydependent, anatomical segregation induced by competition for neurotrophic support. Biol Cybern 75:463-470.

Elliott T, Shadbolt NR (1998) Competition for neurotrophic factors: mathematical analysis. Neural Comput, in press.

Elliott T, Howarth CI, Shadbolt NR (1996) Axonal processes and neural plasticity. I. Ocular dominance columns. Cereb Cortex 6:781-788.

Fields RD, Neale EA, Nelson PG (1990) Effects of patterned electrical activity on neurite outgrowth from mouse neurons. J Neurosci 10:2950-2964.

Galuske RAW, Kim D-S, Castren E, Thoenen H, Singer W (1996) Brain-derived neurotrophic factor reverses experience-dependent synaptic modifications in kitten visual cortex. Eur J Neurosci 8:1554-1559.

Goodhill GJ (1993) Topography and ocular dominance: a model exploring positive correlations. Biol Cybern 69:109-118.

Goodhill GJ, Löwel S (1995) Theory meets experiment: correlated neural activity helps determine ocular dominance column periodicity. Trends Neurosci 18:437-439.

Goodhill GJ, Willshaw DJ (1990) Application of the elastic net algorithm to the formation of ocular dominance stripes. Network 1:41-59.

Goodman LJ, Valverde J, Lim F, Geschwind MD, Federoff HJ, Geller AI, Hefti F (1996) Regulated release and polarized localization of brain-derived neurotrophic factor in hippocampal neurons. Mol Cell Neurosci 7:222-238.

Griesbeck O, Blöchl A, Carnahan JF, Nawa H, Thoenen H (1995) Characterization of brain-derived neurotrophic factor (BDNF) secretion from hippocampal neurons. Soc Neurosci Abstr 21:1046.

Gu Q (1995) Involvement of nerve growth factor in visual cortex plasticity. Rev Neurosci 6:329-351.

Gu Q, Liu Y, Cynader MS (1994) Nerve growth factor-induced ocular dominance plasticity in adult cat visual cortex. Proc Natl Acad Sci USA 91:8408-8412. 
Guillery RW (1972) Binocular competition in the control of geniculate cell growth. J Comp Neurol 144:117-130.

Guillery RW, Stelzner DJ (1970) The differential effects of unilateral lid closure upon the monocular and binocular segments of the dorsal lateral geniculate nucleus in the cat. J Comp Neurol 139:413-422.

Harris AE, Ermentrout GB, Small SL (1997) A model of ocular dominance column development by competition for trophic factor. Proc Natl Acad Sci USA 94:9944-9949.

Hata Y, Stryker MP (1994) Control of thalamocortical afferent rearrangement by postsynaptic activity in developing visual cortex. Science 265:1732-1735.

Hubel DH, Wiesel TN (1962) Receptive fields, binocular interaction and functional architecture in the cat's visual cortex. J Physiol (Lond) 160:106-154.

Hubel DH, Wiesel TN (1965) Binocular interaction in striate cortex of kittens reared with artificial squint. J Neurophysiol 28:1041-1059.

Hubel DH, Wiesel TN (1970) The period of susceptibility to the physiological effects of unilateral eye closure in kittens. J Physiol (Lond) 260:419-436.

LeVay S, Stryker MP, Shatz CJ (1978) Ocular dominance columns and their development in layer IV of the cat's visual cortex: a quantitative study. J Comp Neurol 179:223-244.

LeVay S, Wiesel TN, Hubel DH (1980) The development of ocular dominance columns in normal and visually deprived monkeys. J Comp Neurol 191:1-51.

Lipton SA, Kater SB (1989) Neurotransmitter regulation of neuronal outgrowth, plasticity, and survival. Trends Neurosci 12:265-270.

Löwel S (1994) Ocular dominance column development: strabismus changes the spacing of adjacent columns in cat visual cortex. J Neurosci 14:7451-7468.

Maffei L, Berardi N, Domenici L, Parisi V, Pizzorusso T (1992) Nerve growth factor (NGF) prevents the shift in ocular dominance distribution of visual cortical neurons in monocularly deprived rats. J Neurosci 12:4651-4662.

Mattson MP, Kater SB (1989) Excitatory and inhibitory neurotransmitters in the generation and degeneration of hippocampal neuroarchitecture. Brain Res 478:337-348.

Miller KD (1995) Receptive fields and maps in the visual cortex: models of ocular dominance and orientation columns. In: Models of neural networks III (Domany E, van Hemman JL, Schulten K, eds), pp 55-78. New York: Springer.

Miller KD, Keller JB, Stryker MP (1989) Ocular dominance column development: analysis and simulation. Science 245:605-615.

Montague PR, Gally JA, Edelman GM (1991) Spatial signaling in the development and function of neural connections. Cereb Cortex 1:199-220.

Purves D (1988) Body and brain: a trophic theory of neural connections. Cambridge, MA: Harvard UP.

Reiter HO, Stryker MP (1988) Neural plasticity without postsynaptic action potentials: less-active inputs become dominant when kitten visual cortical cells are pharmacologically inhibited. Proc Natl Acad Sci USA 85:3623-3627.
Reiter HO, Waitzman DM, Stryker MP (1986) Cortical activity blockade prevents ocular dominance plasticity in the kitten visual cortex. Exp Brain Res 65:182-188.

Riddle DR, Lo DC, Katz LC (1995) NT-4-mediated rescue of lateral geniculate neurons from effects of monocular deprivation. Nature 378:189-191.

Salin T, Mudo G, Jiang XH, Timmusk T, Metsis M, Belluardo N (1995) Up-regulation of trkB mRNA expression in the rat striatum after seizures. Neurosci Lett 194:181-184.

Schoups AA, Elliott RC, Friedman WJ, Black IB (1995) NGF and BDNF are differentially modulated by visual experience in the developing geniculocortical pathway. Dev Brain Res 86:326-334.

Schuster CM, Davis GW, Fetter RD, Goodman CS (1996a) Genetic dissection of structural and functional components of synaptic plasticity. I. Fasciclin II controls synaptic stabilization and growth. Neuron 17:641-654.

Schuster CM, Davis GW, Fetter RD, Goodman CS (1996b) Genetic dissection of structural and functional components of synaptic plasticity. II. Fasciclin II controls presynaptic structural plasticity. Neuron 17:655-667.

Shatz CJ, Lindström S, Wiesel TN (1977) The distribution of afferents representing the right and left eyes in the cat's visual cortex. Brain Res 131:103-116.

Stryker MP, Harris W (1986) Binocular impulse blockade prevents the formation of ocular dominance columns in cat visual cortex. J Neurosci 6:2117-2133.

Stryker MP, Strickland SL (1984) Physiological segregation of ocular dominance columns depends on the pattern of afferent electrical activity. Invest Ophthalmol Vis Sci [Suppl] 25:278.

Sussdorf WS, Campenot RB (1986) Influence of the extracellular potassium environment on neurite growth in sensory neurons, spinal cord neurons and sympathetic neurons. Dev Brain Res 25:43-52.

Swindale NV (1980) A model for the formation of ocular dominance stripes. Proc R Soc Lond [Biol] 208:243-264.

Thoenen H (1995) Neurotrophins and neuronal plasticity. Science 270:593-598.

Tieman SB, Tumosa N (1997) Alternating monocular exposure increases the spacing of ocularity domains in area 17 of cats. Vis Neurosci 14:929-938.

Turrigiano GG, Leslie KR, Desai NS, Rutherford LC, Nelson SB (1998) Activity-dependent scaling of quantal amplitude in neocortical neurons. Nature 391:892-896.

Weliky M, Katz LC (1997) Disruption of orientation tuning in visual cortex by artificially correlated neuronal activity. Nature 386:680-685.

Yan HQ, Mazow ML, Dafny N (1996) NGF prevents the changes induced by monocular deprivation during the critical period in rats. Brain Res 706:318-322.

Zafra F, Castren E, Thoenen H, Lindholm D (1991) Interplay between glutamate and $\gamma$-aminobutyric acid transmitter systems in the physiological regulation of brain-derived neurotrophic factor and nerve growth factor synthesis in hippocampal neurons. Proc Natl Acad Sci USA 88:10037-10041. 